\title{
Ultrasonic Studies on Molecular Interactions in Binary Mixtures of $N$-Methyl Aniline with Methyl Isobutylketone, +3-Pentanone, and +Cycloalkanones at $303.15 \mathrm{~K}$
}

\author{
M. Gowrisankar • P. Venkateswarlu $\cdot$ K. Sivakumar $\cdot$ S. Sivarambabu
}

Received: 15 April 2012/Accepted: 3 September 2012/Published online: 25 May 2013

(C) The Author(s) 2013. This article is published with open access at Springerlink.com

\begin{abstract}
Densities, $\rho$, viscosities, $\eta$, and ultrasonic sound velocities $u$ of pure methyl isobutylketone, diethylketone, cyclopentanone, cyclohexanone, 2-methyl cyclohexanone and those of their binary mixtures with $N$-methyl aniline were measured at $303.15 \mathrm{~K}$ over the entire composition range. These experimental data have been used to calculate the excess volume $\left(V^{\mathrm{E}}\right)$, deviation in ultrasonic sound velocity $(\Delta u)$, isentropic compressibility $\left(\kappa_{s}\right)$, intermolecular free length $\left(L_{\mathrm{f}}\right)$, excess intermolecular free length $\left(L_{\mathrm{f}}^{\mathrm{E}}\right)$, acoustic impedance $(Z)$, excess isentropic compressibility $\left(\kappa_{S}^{\mathrm{E}}\right)$, deviation in viscosity $(\Delta \eta)$ and excess Gibbs energy of activation of viscous flow $\left(G^{* \mathrm{E}}\right)$. The viscosity data have been correlated using three equations proposed by Grunberg and Nissan, Katti and Chaudhri, and Hind et al. The excess/deviations have been fitted by Redlich-Kister equation and the results are discussed in terms of molecular interactions present in these mixtures.
\end{abstract}

Keywords Ultrasonic speed $\cdot$ Viscosity $\cdot$ Ketone $\cdot$ Excess volume $\cdot$ Intermolecular interaction $\cdot N$-methyl aniline

\section{Introduction}

The study of thermodynamic properties of binary liquid mixtures has proved to be a useful tool in elucidating the interactions that are operating between component molecules [1].

\author{
M. Gowrisankar \\ Department of Chemistry, J.K.C. College, Guntur 522006, India \\ P. Venkateswarlu \\ Department of Chemistry, S.V. University, Tirupathi 517502, India
}

K. Sivakumar

Department of Chemistry, S.V. Arts U.G. \& P.G. College (T.T.D’S), Tirupathi 517502, India

S. Sivarambabu $(\bowtie)$

Research Supervisor in Chemistry, Acharya Nagarjuna University, Guntur, India e-mail: sivarambabu99@gmail.com; sivakumarkasi64@gmail.com 
Excess thermodynamic functions, which depend on the composition, temperature and pressure of the system, are of great importance to a chemical engineer in the design of industrial separation process and to a chemist for arriving at theories of liquid mixtures. Accurate knowledge of thermodynamic properties of organic liquid mixtures has relevance in understanding the molecular interactions between the components of the mixture.

The primary objective is to measure the speeds of sound and densities of liquid systems in order to estimate the value of isentropic compressibility, which in turn is widely used to study the molecular interactions through its excess value. The experimental data in the present investigation, namely density, ultrasonic sound velocity and viscosity, were used to compute the thermodynamic functions such as excess molar volume $\left(V^{\mathrm{E}}\right)$, deviations in ultrasonic speed $(\Delta u)$, isentropic compressibility $\left(\kappa_{s}\right)$, excess isentropic compressibility $\left(\kappa_{S}^{\mathrm{E}}\right)$, intermolecular free length $\left(L_{\mathrm{f}}\right)$, excess intermolecular free length $\left(L_{\mathrm{f}}^{\mathrm{E}}\right)$, acoustic impedance $(Z)$, deviation in viscosity $(\Delta \eta)$ and excess Gibbs energy of activation of viscous flow $\left(G^{* \mathrm{E}}\right)$. In principle, the interactions between component molecules can be derived from a study of deviations from the ideal behavior of properties like molar volume, compressibility and viscosity. The sign of deviation of an excess property may be negative or positive from the ideal value depending on the type and extent of interactions between unlike molecules. In the present study, the density, ultrasonic sound velocity and viscosity of pure $N$-methyl aniline (N-MA), methyl isobutylketone (MIBK), diethylketone (DEK), cyclohexanone (CH), 2-methylcyclohexanone and their mixtures were measured at $303.15 \mathrm{~K}$ over the entire composition range. By using this experimental data, various thermodynamic functions were calculated. Further, the experimental viscosity data were used to test the capability of semiempirical relations of Grunberg and Nissan [2], Katti and Chaudhri [3] and Hind et al. [4]. The present work was under taken to study the effect of sign and magnitude of excess volume, excess isentropic compressibility and deviation in viscosity when $N$-methyl aniline is mixed with various aliphatic ketones. The organic liquids used in the present study are important due to their industrial applications. $\mathrm{N}$-methyl aniline is used as an intermediate of manufactured dyes, agrochemicals and in preparation of some organic compounds. Ketones are used as solvents, polymer precursors and in the preparation of many pharmaceuticals.

We reported in our earlier communications $[5,6]$ excess volumes, sound velocities and viscosities for the binary mixtures of $\mathrm{N}$-ethyl aniline and $\mathrm{N}, \mathrm{N}$-dimethyl aniline with aromatic ketones. A survey of literature has shown that thermodynamic properties of amines with 2-propanone, 2-butanone and 2-pentanone were reported [7-9]. As far we are aware no experimental excess molar volume, ultrasonic sound velocity and viscosity data of for the present systems under investigation have been reported in the literature, Hence, the present work was undertaken to study the effect of position of the carbonyl group in a ketone molecule and chain length of ketonic molecules.

\section{Experimental}

All the chemicals used in the present work were of Analytical Reagent (AR) grade (S.D. Fine Chemicals Ltd., India) purified as described in the literature [10]. The pure samples were attained by fractional distillation and the purity of chemicals were checked by comparing the measured densities, ultrasonic sound velocity viscosity and heat capacities, which are in good agreement with literature values [10-19] and these results are given in Table 1. The purities of the samples were further confirmed by GLC, which showed single sharp peaks. Before use, the chemicals were stored over $0.4 \mathrm{~nm}$ molecular sieves for about 


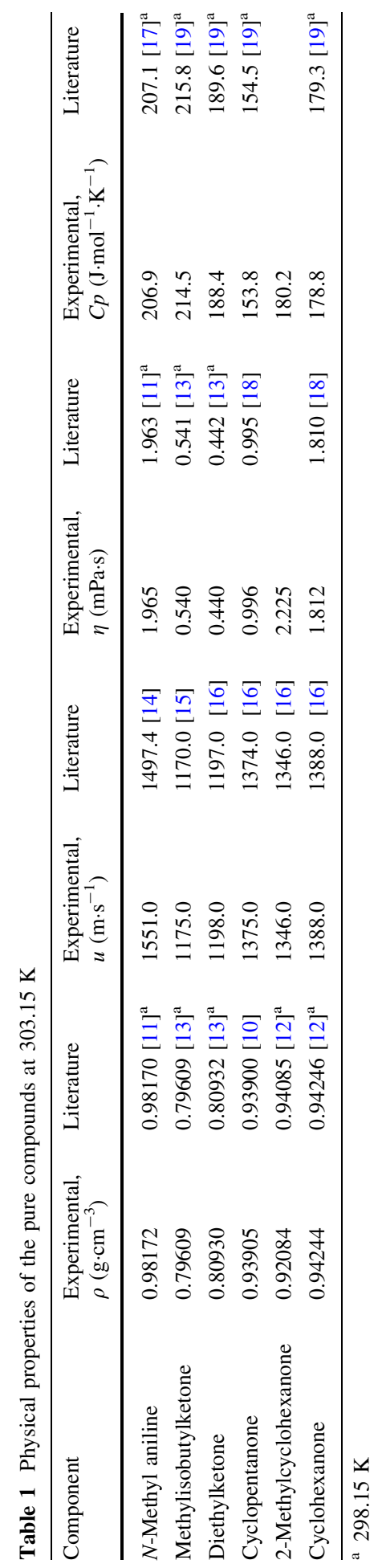


$72 \mathrm{~h}$ to remove water and were later degassed. The binary mixtures of $N$-methyl aniline with methylisobutylketone, diethylketone, cyclopentanone (CP), cyclohexanone and 2-methylcyclohexanone were prepared in glass bottles with air tight stoppers and adequate precautions were taken to minimize losses through evaporation. The weighing of solutions was done using an Acculab ALC-210.4 digital electronic balance with an uncertainty of $\pm 0.0001 \mathrm{~g}$. The uncertainty in solution composition, expressed in mole fraction, was found to be less than $5 \times 10^{-4}$.

After mixing the sample, the bubble-free homogeneous sample was transferred into the U-tube of the densimeter using a syringe. The density measurements were performed with a Rudolph Research Analytical digital densimeter (DDH-2911 Model), equipped with a built- in solid-state thermostat and a resident program, at the temperature $303.15 \pm 0.03 \mathrm{~K}$. Typically, density precisions are $\pm 0.00005 \mathrm{~g} \cdot \mathrm{cm}^{-3}$. Proper calibration at each temperature was achieved with doubly distilled, deionized water and with air as standards. A multi frequency ultrasonic interferometer (M-82 Model, Mittal Enterprise, New Delhi, India), operated at $2 \mathrm{MHz}$, was used to measure the ultrasonic velocities in the binary liquid mixtures at the constant temperature $303.15 \mathrm{~K}$ controlled by a digital constant temperature water bath. The uncertainty in the measurement of ultrasonic sound velocity is $\pm 0.2 \%$. The temperature stability is maintained within $\pm 0.02 \mathrm{~K}$ by circulating thermostated water around the cell with a circulating pump. In order to minimize the uncertainty of the measurement, several maxima are allowed to pass and their number, 50 in the present study, is counted. All maxima are recorded with the highest swing of the needle on the micrometer scale. The total distance $d(\mathrm{~cm})$ moved by the reflector is given by $d=n \lambda / 2$, where $\lambda$ is the wave length. The frequency, $v$, of the crystal being accurately known $(2.0 \mathrm{MHz})$, the speed of sound, $u$ in $\mathrm{m} \cdot \mathrm{s}^{-1}$ is calculated by using the relation $u=v \lambda$. The working of the interferometer was tested by making measurements for pure samples of benzene, toluene, chloroform, chlorobenzene, and acetone and the measured sound velocities of these liquids were in good agreement with those reported in the literature [20]. The viscosities of the pure liquids and their binary mixtures were measured by using a modified Ubbelohde capillary type viscometer [21]. The viscometer was calibrated with pure water and the liquid was allowed to stand for about $30 \mathrm{~min}$ in a thermostatic water bath so that thermal fluctuations in the viscometer were minimized. The accuracy in viscosity data is $\pm 0.005 \mathrm{~m} \cdot \mathrm{Pa} \cdot \mathrm{s}$

\section{Theory and Calculations}

The experimental values of density $\rho$, viscosity $\eta$ and ultrasonic velocity $u$, of pure liquids and their mixtures as function of mole fraction of $N$-methyl aniline at $303.15 \mathrm{~K}$ are given in Table 2. The derived parameters such as $\kappa_{s}, L_{\mathrm{f}}$, and $Z$ were calculated using the following relations

$$
\begin{gathered}
\kappa_{s}=u^{-2} \rho^{-1} \\
L_{\mathrm{f}}=K / u \rho^{1 / 2} \\
Z=u \rho
\end{gathered}
$$

In the above equations, $\rho$ is the density and $u$ is the ultrasonic speed of the solutions. $K$ is a temperature dependent constant [22]. The deviations in excess functions from ideality provide a relatively better tool to assess the strength of interaction between the component molecules of the binary mixtures. $V^{\mathrm{E}}, \kappa_{S}^{\mathrm{E}} \Delta \eta, \Delta u$ and $L_{\mathrm{f}}^{\mathrm{E}}$ were calculated from experimental data using the following expressions: 


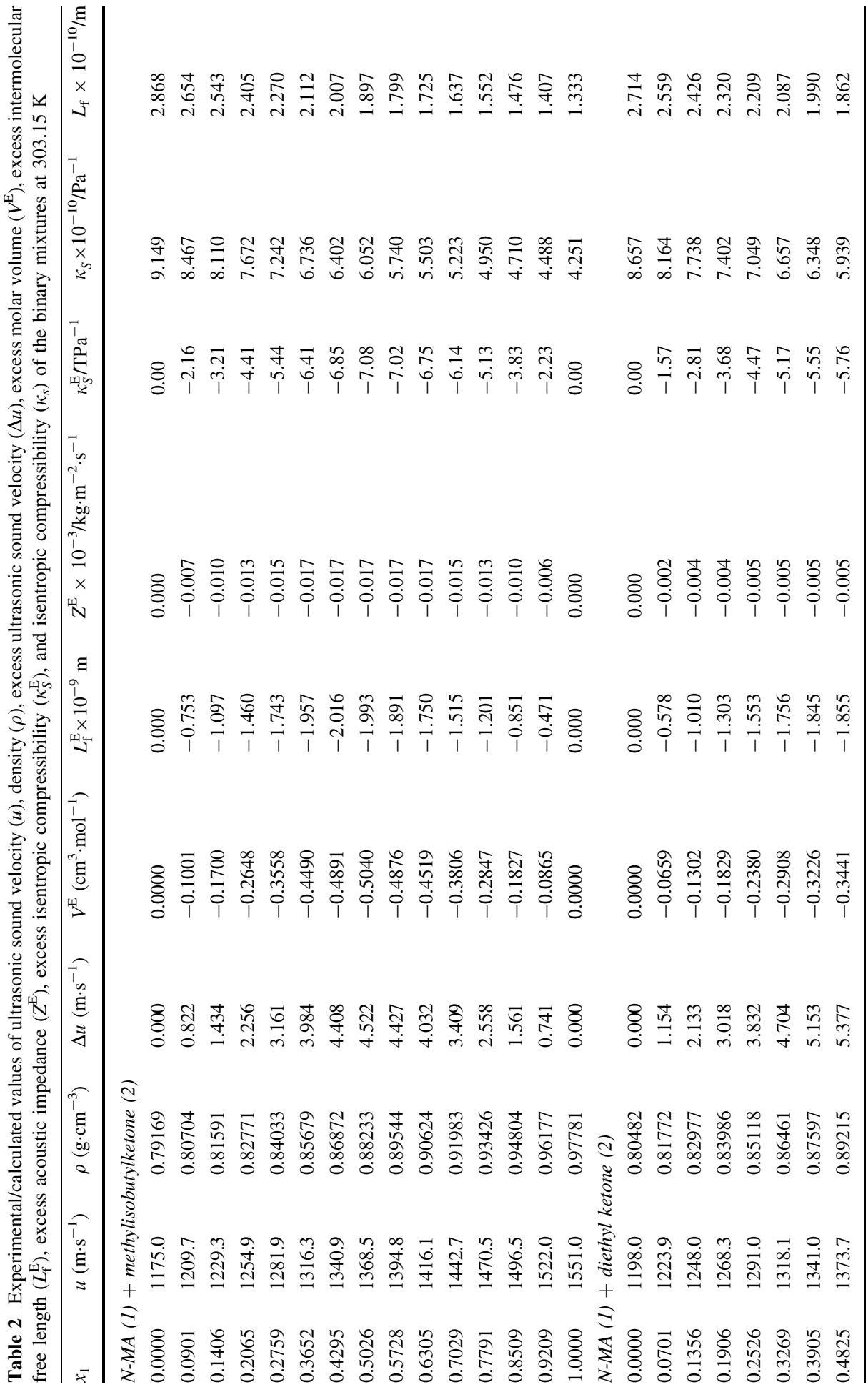




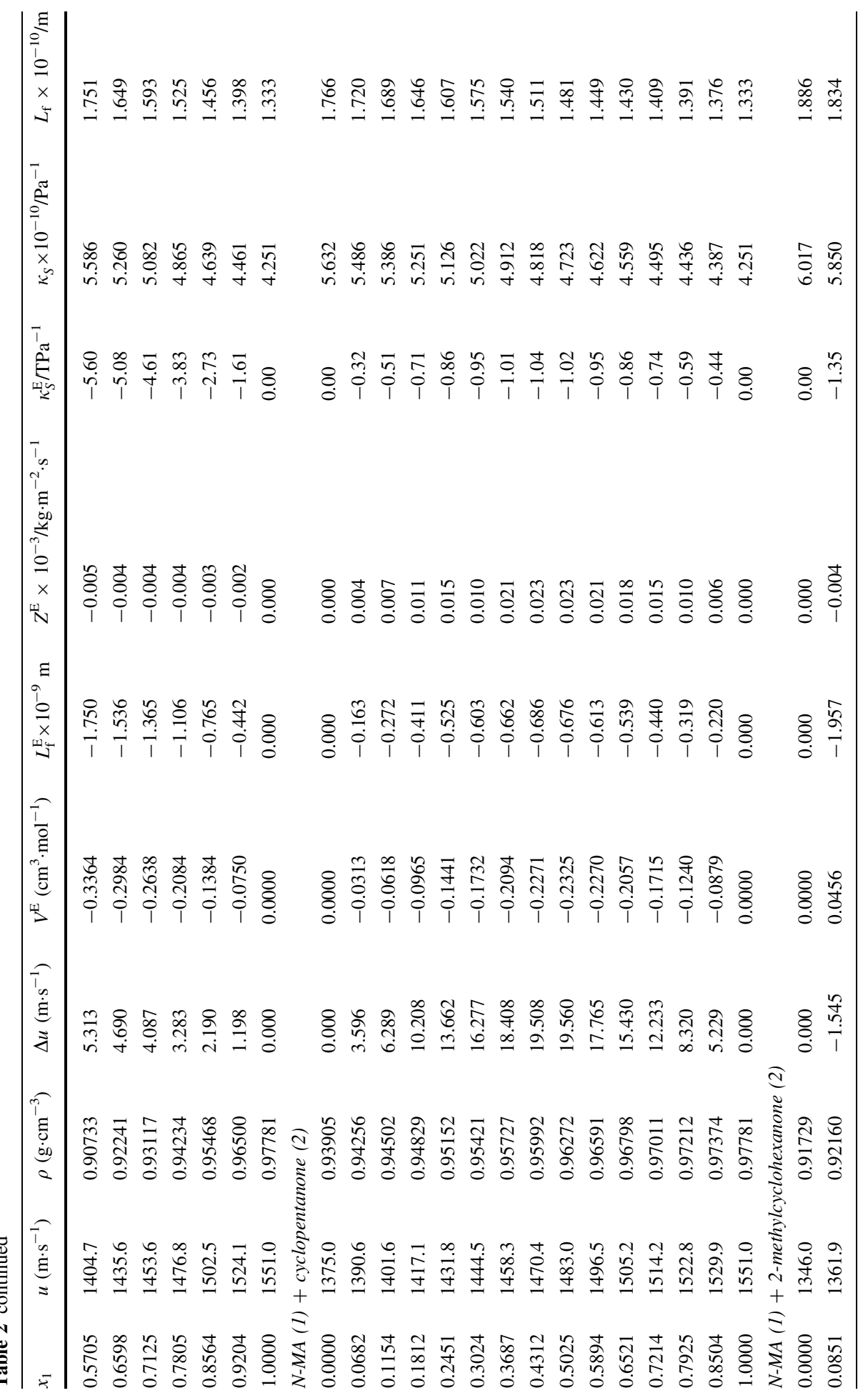




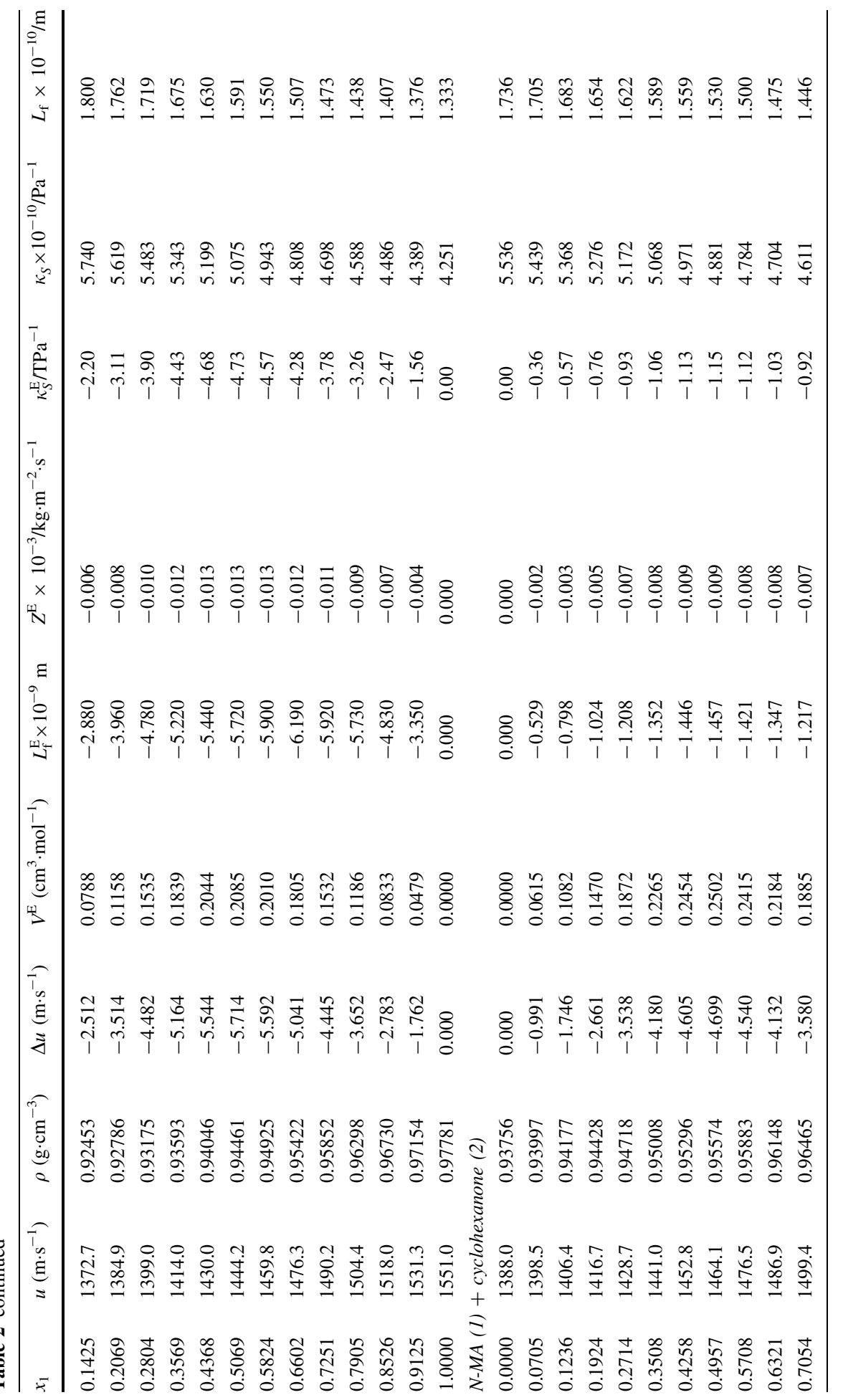




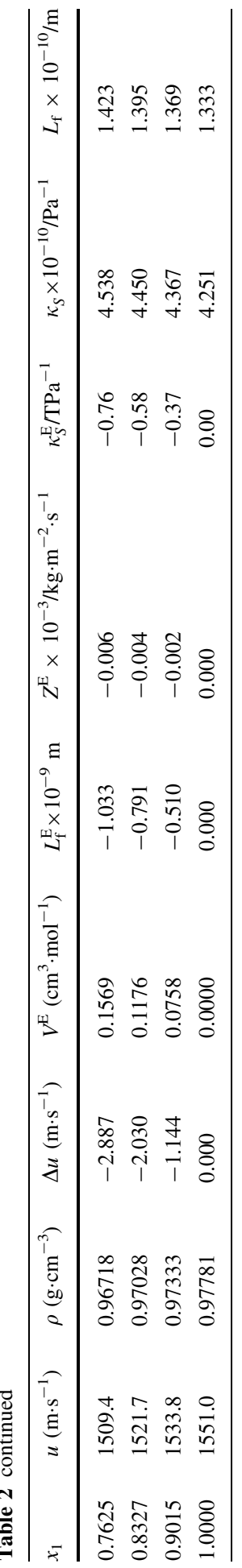




$$
\begin{gathered}
V^{\mathrm{E}}=\frac{x_{1} M_{1}+x_{2} M_{2}}{\rho_{\mathrm{m}}}-\left\{\frac{x_{1} M_{1}}{\rho_{1}}+\frac{x_{2} M_{2}}{\rho_{2}}\right\} \\
\kappa_{S}^{\mathrm{E}}=\kappa_{S}-\kappa_{S}^{\mathrm{id}} \\
\Delta \eta=\eta-\left(x_{1} \eta_{1}+x_{2} \eta_{2}\right) \\
\Delta u=u-\left(x_{1} u_{1}+x_{2} u_{2}\right) \\
L_{f}^{\mathrm{E}}=L_{f}-\left(x_{1} L_{f 1}+x_{2} L_{f 2}\right)
\end{gathered}
$$

In the above equations, $M_{i}, \eta_{i}, u_{i}$ and $\rho_{i}$ represent the molecular weight, isentropic compressibility, viscosity, ultrasonic velocity and density of component $i$ and $\rho_{\mathrm{m}}, \kappa_{S}, \eta$, and $u$ the corresponding values of the mixture.

The excess isentropic compressibilities $\left(\kappa_{S}^{\mathrm{E}}\right)$ [23] for the binary mixtures were calculated using the relations:

$$
\kappa_{S}^{\mathrm{E}}=\kappa_{S}-\kappa_{S}^{\mathrm{id}}
$$

where $\kappa_{S}^{\text {id }}$ was calculated from the relation:

$$
\kappa_{S}^{\mathrm{id}}=\sum_{i=1}^{2} \phi_{i}\left(\kappa_{S i}+\frac{T V_{i} \alpha_{i}^{2}}{C_{p i}}\right)-\frac{T\left(\sum_{i=1}^{2} x_{i} V_{i}\right)\left(\sum_{i=1}^{2} \phi_{i} \alpha_{i}\right)}{\sum_{i=1}^{2} x_{i} C_{p i}}
$$

where $\phi_{i}$ is the ideal state volume fraction and is defined by the relation:

$$
\phi_{i}=\frac{x_{i} V_{i}}{\sum_{i=1}^{2} x_{i} V_{i}}
$$

The variation of $V^{\mathrm{E}}, \kappa_{S}^{\mathrm{E}}, \Delta \eta$ and $\Delta u$ with mole fraction were fitted to the Redlich-Kister equation [24] of the type:

$$
Y^{\mathrm{E}}=x_{1} x_{2}\left\{a_{0}+a_{1}\left(x_{1}-x_{2}\right)+a_{2}\left(x_{1}-x_{2}\right)^{2}\right\}
$$

where $Y^{\mathrm{E}}$ is $V^{\mathrm{E}}, \Delta u, \kappa_{S}^{\mathrm{E}}$ or $\Delta \eta$. The values of $a_{0}, a_{1}$ and $a_{2}$ are the coefficients of the polynomial equation and were obtained by the method of least-squares and are given in Table 3 along with standard deviation values at $303.15 \mathrm{~K}$. The standard deviations are calculated by using the equation:

$$
\sigma\left(Y^{\mathrm{E}}\right)=\left\{\frac{\sum_{i=1}^{n}\left(Y_{\mathrm{obs}}^{\mathrm{E}}-Y_{\mathrm{cal}}^{\mathrm{E}}\right)^{2}}{n-m}\right\}^{1 / 2}
$$

where $n$ is the total number of experimental points and $m$ is the number of coefficients. The excess Gibbs energy of activation of viscous flow $\left(G^{*}\right)$ is obtained by the equation:

$$
G^{* \mathrm{E}}=R T\left(\ln \eta V-\sum x_{i} \ln \eta_{i} V_{i}\right)
$$


Table 3 Coefficients of the Redlich-Kister equation and standard deviation values at $303.15 \mathrm{~K}$

\begin{tabular}{|c|c|c|c|c|c|}
\hline Binary mixtures & Functions & $a_{0}$ & $a_{1}$ & $a_{2}$ & $\sigma$ \\
\hline \multirow[t]{4}{*}{$\mathrm{N}-\mathrm{MA}+$ cyclopentanone } & $V^{\mathrm{E}} \mathrm{cm}^{3} \cdot \mathrm{mol}^{-1}$ & -0.938 & -0.037 & 0.557 & 0.003 \\
\hline & $\Delta u \mathrm{~m} \cdot \mathrm{s}^{-1}$ & 78.054 & -17.532 & -49.86 & 0.042 \\
\hline & $\kappa_{S}^{\mathrm{E}} \mathrm{TPa}^{-1}$ & -40.807 & 10.036 & -1.908 & 0.021 \\
\hline & $\Delta \eta \mathrm{mPa} \cdot \mathrm{s}$ & -0.786 & 0.064 & 0.618 & 0.001 \\
\hline \multirow[t]{4}{*}{$\mathrm{N}-\mathrm{MA}+$ methylcyclohexanone } & $V^{\mathrm{E}} \mathrm{cm}^{3} \cdot \mathrm{mol}^{-1}$ & 0.835 & 0.008 & -0.356 & 0.001 \\
\hline & $\Delta u / \mathrm{m} \cdot \mathrm{s}^{-1}$ & -22.771 & -0.927 & 2.748 & 0.050 \\
\hline & $\kappa_{S}^{\mathrm{E}} \mathrm{TPa}^{-1}$ & -49.195 & -3.018 & -0.184 & 0.026 \\
\hline & $\Delta \eta \mathrm{mPa} \cdot \mathrm{s}$ & -0.647 & 0.018 & 0.497 & 0.003 \\
\hline \multirow[t]{4}{*}{ N-MA + cyclohexanone } & $V^{\mathrm{E}} \mathrm{cm}^{3} \cdot \mathrm{mol}^{-1}$ & 0.976 & -0.080 & -0.136 & 0.005 \\
\hline & $\Delta u \mathrm{~m} \cdot \mathrm{s}^{-1}$ & -18.744 & 1.615 & 6.897 & 0.032 \\
\hline & $\kappa_{S}^{\mathrm{E}} \mathrm{TPa}^{-1}$ & -54.630 & 0.817 & 0.047 & 0.030 \\
\hline & $\Delta \eta \mathrm{mPa} \cdot \mathrm{s}$ & 0.107 & -0.002 & -0.079 & 0.002 \\
\hline \multirow[t]{4}{*}{$\mathrm{N}$-MA + 4-methyl-2-pentanone } & $V^{\mathrm{E}} \mathrm{cm}^{3} \cdot \mathrm{mol}^{-1}$ & -2.019 & -0.002 & 1.181 & 0.001 \\
\hline & $\Delta u \mathrm{~m} \cdot \mathrm{s}^{-1}$ & 18.173 & 0.296 & -11.79 & 0.027 \\
\hline & $\kappa_{S}^{\mathrm{E}} \mathrm{TPa}^{-1}$ & -283.168 & -25.508 & -1.960 & 0.030 \\
\hline & $\Delta \eta \mathrm{mPa} \cdot \mathrm{s}$ & 0.148 & 0.001 & -0.021 & 0.001 \\
\hline \multirow[t]{4}{*}{$\mathrm{N}$-MA + 3-pentanone } & $V^{\mathrm{E}} \mathrm{cm}^{3} \cdot \mathrm{mol}^{-1}$ & -1.380 & 0.001 & 0.504 & 0.001 \\
\hline & $\Delta u \mathrm{~m} \cdot \mathrm{s}^{-1}$ & 21.709 & -0.862 & -6.918 & 0.044 \\
\hline & $\kappa_{S}^{\mathrm{E}} \mathrm{TPa}^{-1}$ & -99.322 & -0.838 & -0.008 & 0.029 \\
\hline & $\Delta \eta \mathrm{mPa} \cdot \mathrm{s}$ & 0.222 & -0.007 & -0.016 & 0.001 \\
\hline
\end{tabular}

where $V_{i}$ and $V$ are the molar volumes of the component $i$ and molar volume of the mixture respectively; $R$ and $T$ have their usual meanings. Grunberg and Nissan [2] proposed the following equation for the measurement of viscosity of liquid mixtures:

$$
\ln \eta=x_{1} \ln \eta_{1}+x_{2} \ln \eta_{2}+x_{1} x_{2} d
$$

where $d$ is a parameter proportional to the interchange energy, which reflects the nonideality of the system. Katti and Chaudhri [3] proposed the following equation:

$$
\ln \eta V=x_{1} \ln \eta_{1} V_{1}+x_{2} \ln \eta_{2} V_{2}+x_{1} x_{2} \frac{W_{\text {vis }}}{R T}
$$

where $W_{\text {vis }} / R T$ is an interaction term. Hind et al. [4] suggested an equation for the viscosity of binary liquid mixtures as:

$$
\eta=x_{1}^{2} \eta_{1}+x_{2}^{2} \eta_{2}+2 x_{1} x_{2} H_{12}
$$

where $H_{12}$ is the Hind interaction parameter.

\section{Results and Discussion}

Ultrasound waves are high frequency mechanical waves. Their velocities in a medium depend inversely on the density and compressibility of the medium. The variation of ultrasonic velocity in a mixture depends upon the increase or decrease of intermolecular 
free length $\left(L_{f}\right)$ after mixing the components the computed $L_{\mathrm{f}}^{\mathrm{E}}$ and is graphically represented in Figs. 1, 2, 3 and 4. Further, $\left(\kappa_{S}^{\mathrm{E}}\right)$ values are calculated as described [23] are also graphically represented in Figs. 5 and 6 for binary mixtures of $N$-methylaniline with all ketones. Generally, negative values of $\Delta u$ indicate dispersion forces due to weak interactions whereas positive values of $\Delta u$ indicate strong interactions $[25,26]$. The sign and magnitude of $\Delta u$ play important roles in describing molecular rearrangements among the component molecules in the mixtures which reflect intermolecular interactions between the molecules. A perusal of data in Table 2 shows that the values of $\Delta u$ are positive for N-MA + DEK, N-MA + MIBK, and N-MA + CP, whereas those for the mixtures of N-MA + CH and N-MA + Me-CH are negative over the entire composition ranges at $303.15 \mathrm{~K}$.

An examination of data in Table 2 shows that the excess isentropic compressibility $\left(\kappa_{S}^{\mathrm{E}}\right)$ and excess intermolecular free length $\left(L_{\mathrm{f}}^{\mathrm{E}}\right)$ are negative in all of the binary systems over the entire range of composition. According to Sri Devi et al. [27], negative excess values are due to closely packed molecules which accounts for the existence of strong molecular interactions, whereas positive excess values reflect weak interactions between unlike molecules. The sign of the excess isentropic compressibility $\left(\kappa_{S}^{\mathrm{E}}\right)$ and excess intermolecular free length $\left(L_{\mathrm{f}}^{\mathrm{E}}\right)$ are useful in assessing the compaction due to molecular interactions in liquid mixtures through: hydrogen-bonding, charge transfer, dipole-dipole and dipole-induced dipole interactions, interstitial accommodation and orientational ordering [28], which lead to a more compact structure, leading to negative values of the excess isentropic compressibility and excess intermolecular free length. Hence negative values of the excess isentropic compressibility $\left(\kappa_{S}^{\mathrm{E}}\right)$ and excess intermolecular free length $\left(L_{\mathrm{f}}^{\mathrm{E}}\right)$ in the present systems suggests that strong molecular interactions are present between unlike molecules in the liquid mixtures.

The $\kappa_{S}^{\mathrm{E}}$ values for the systems containing methyl isobutyl ketone, diethyl ketone and alicyclic ketones fall in the orders: methyl isobutyl ketone $>$ diethyl ketone and cyclopentanone $>2$-methylcyclohexanone $>$ cyclohexanone.

The order of alicyclic ketones is cyclopentanone $>2$-methylcyclohexanone $>$ cyclohexanone which suggests that an increase in cyclic structure hinders the interaction. The

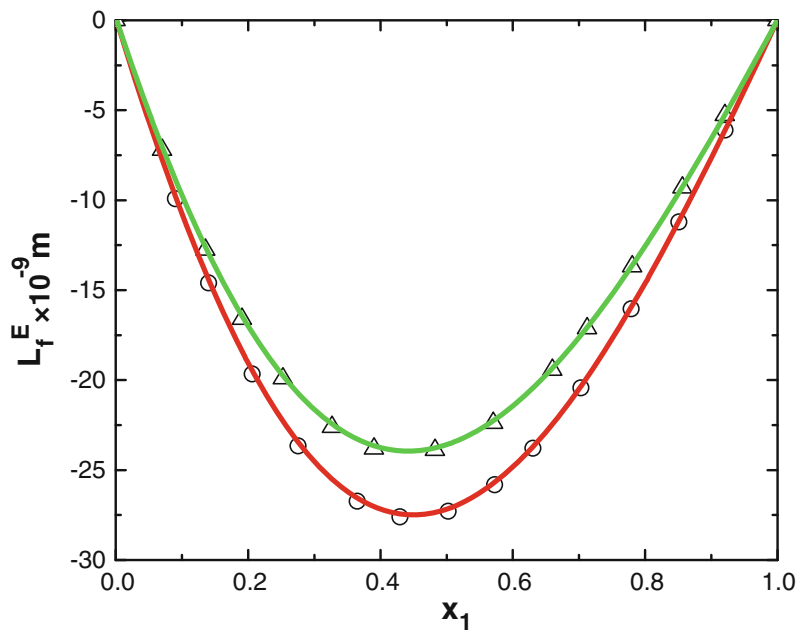

Fig. 1 Variation of the excess intermolecular free length $\left(L_{\mathrm{f}}^{\mathrm{E}}\right)$ with mole fraction $\left(x_{1}\right)$ of N-MA in the binary liquid mixtures of N-MA with methyl isobutyl ketone (small circle) and diethyl ketone (small triangle) at $303.15 \mathrm{~K}$ 
Fig. 2 Variation of the excess intermolecular free length $\left(L_{\mathrm{f}}^{\mathrm{E}}\right)$ with mole fraction $\left(x_{1}\right)$ of N-MA in the binary liquid mixtures of N-MA with cyclopentanone (small circle), 2-methyl cyclohexanone (small triangle) and cyclohexanone (small black circle) at $303.15 \mathrm{~K}$

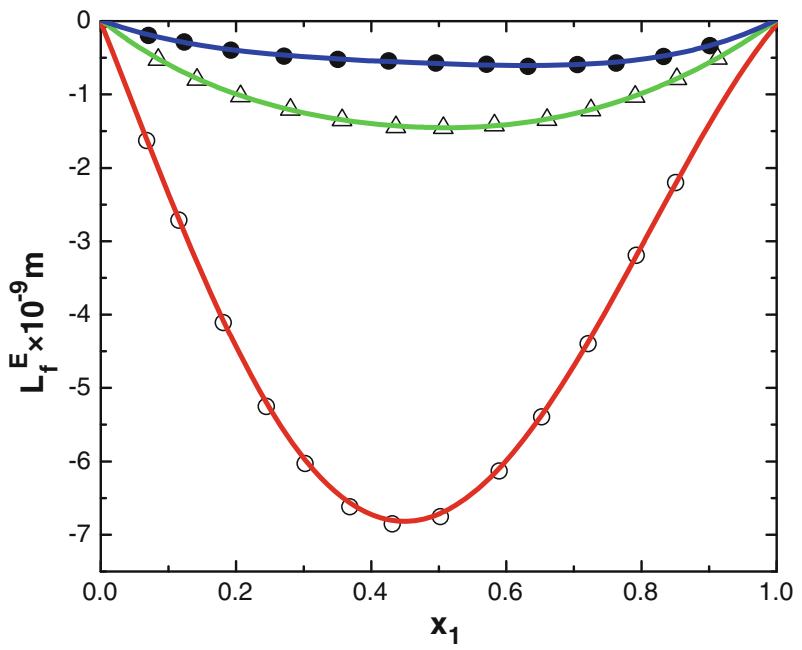

Fig. 3 Deviation of ultrasonic speed $\Delta u$ with mole fraction $\left(x_{1}\right)$ of N-MA in the binary liquid mixtures of N-MA with methyl isobutyl ketone (small circle) and diethyl ketone (small triangle), at $303.15 \mathrm{~K}$

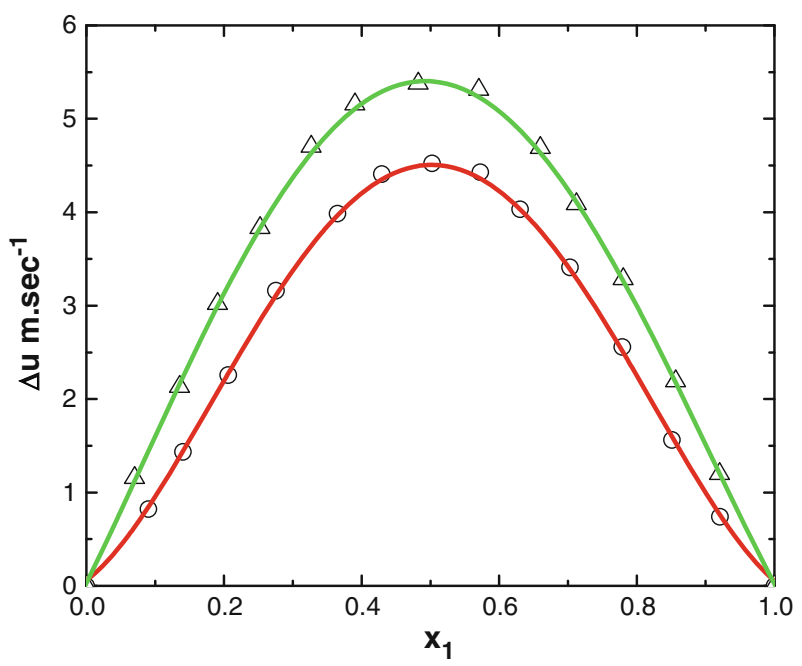

higher values for 2-methylcyclohexanone solutions compared to cyclohexanone may be due to the presence of the methyl group which increases the negative charge on the oxygen atom of the carbonyl group [16]. Hence, the above order may be justified, suggesting that dipole-dipole interactions between unlike molecules are prevailing [29].

A careful study of data in the Table 2 suggests that the excess volume data for the systems N-MA + MIBK, N-MA + DEK, and N-MA + CP are negative whereas for the mixtures of $\mathrm{N}-\mathrm{MA}+\mathrm{CH}, \mathrm{N}-\mathrm{MA}+\mathrm{Me}-\mathrm{CH}$ are positive over the entire composition ranges at $303.15 \mathrm{~K}$. The excess volume data of the binary systems of $N$-methylaniline with ketones are graphically represented in Figs. 7 and 8, it can be explained qualitatively by taking into consideration the following factors: (1) mutual loss of dipolar association due to addition of the second component and contributions due to difference in size and shape of the components, and (2) dipole-dipole and dipole-induced dipole interaction between 
Fig. 4 Deviation of ultrasonic speed $\Delta u$ with mole fraction $\left(x_{1}\right)$ of N-MA in the binary liquid mixtures of N-MA with cyclopentanone (small circle), 2-methyl cyclohexanone (small triangle) and cyclohexanone (small black circle) at $303.15 \mathrm{~K}$

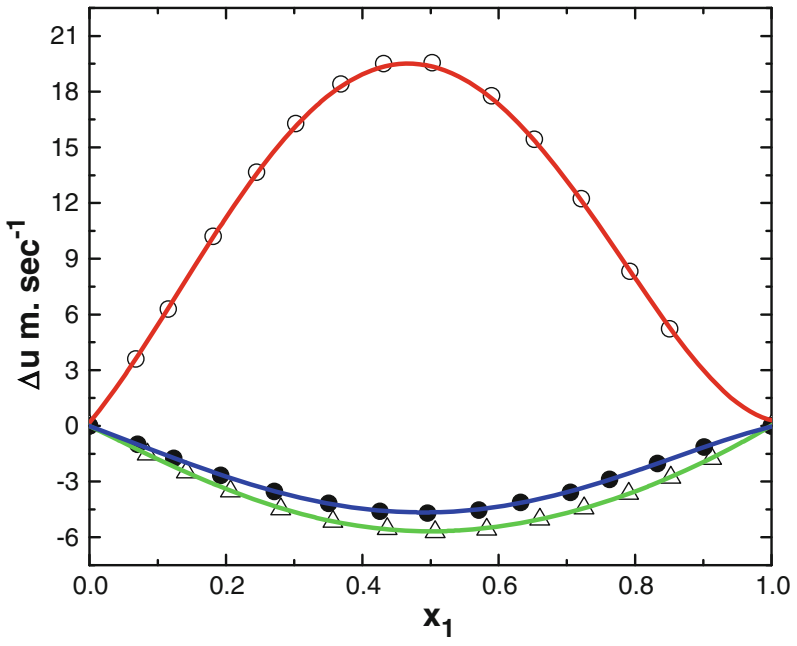

Fig. 5 Excess of isentropic compressibility $\kappa_{S}^{\mathrm{E}}$ with mole fraction $\left(x_{1}\right)$ of N-MA in the binary liquid mixture of N-MA with methyl isobutyl ketone (small circle) and diethyl ketone (small triangle), at $303.15 \mathrm{~K}$

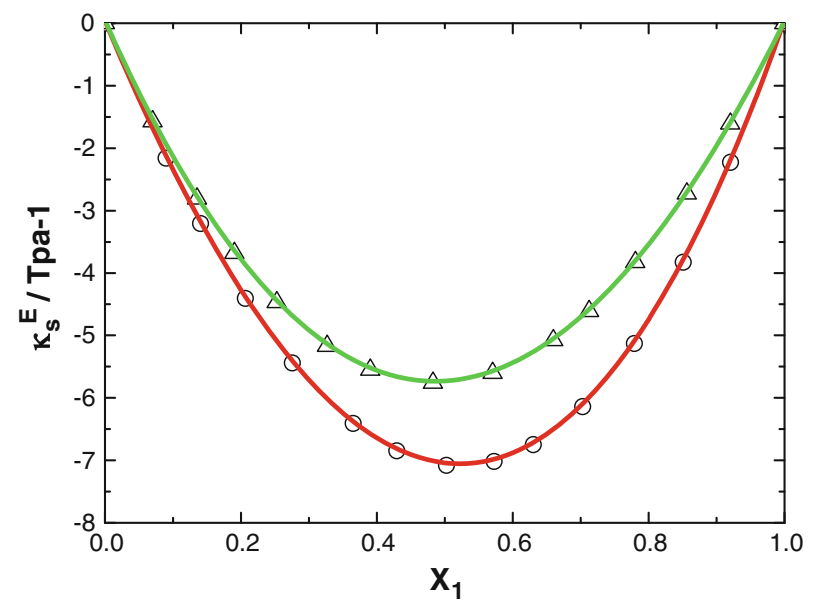

unlike molecules, formation of H-bonds, and interstitial accommodation of the smaller molecules into voids created by the larger molecules due to the difference in molar volumes. The first factor contributes to expansion and the latter factors lead to a decrease in volume. The experimental results in the present investigation suggest that the factors responsible for contraction in volume are dominant over the entire composition range in the mixtures $\mathrm{N}$-methyl aniline with methyl isobutyl ketone, diethyl ketone and cyclopentanone. Furthermore, the observed negative values show that there exists dipole-dipole interactions between unlike molecules and also the formation of hydrogen bonds between the oxygen atom of the carbonyl group of the ketones and the hydrogen atom of the amino group of $N$-methyl aniline. Further, $N$-methyl aniline acts as a proton acceptor and forms strong hydrogen bonds with aliphatic ketones. This hypothesis is substantiated by the considerable contraction in volume that is observed in the mixtures of $N$-methyl aniline with aliphatic ketones. The more negative $V^{\mathrm{E}}$ values for the system methyl isobutyl ketone 
Fig. 6 Excess isentropic compressibility $\kappa_{S}^{\mathrm{E}}$ with mole fraction $\left(x_{1}\right)$ of N-MA in the binary liquid mixture of N-MA with cyclopentanone (small circle), cyclohexanone (small triangle) and 2-methyl cyclohexanone (small black circle) at $303.15 \mathrm{~K}$

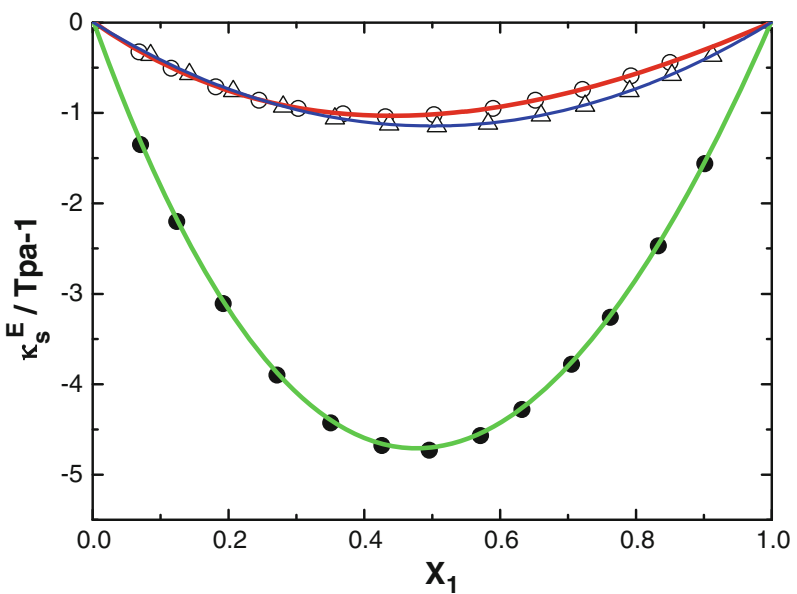

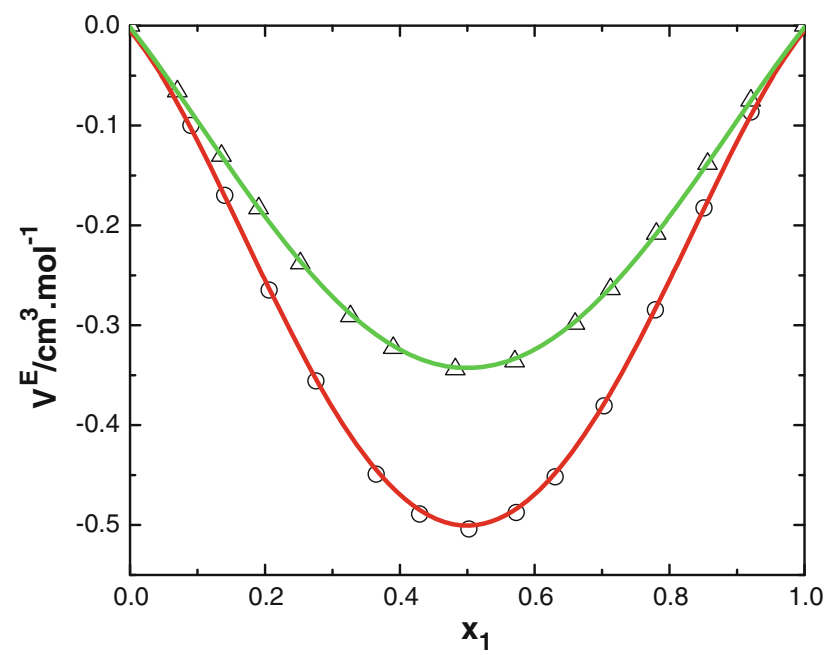

Fig. 7 Variation of excess molar volume $\left(V^{\mathrm{E}}\right)$ with mole fraction $\left(x_{1}\right)$ of N-MA in the binary liquid mixtures of N-MA with methyl isobutyl ketone (small circle) and diethyl ketone (small triangle) at $303.15 \mathrm{~K}$

may be ascribed to the presence of the methyl group on the third carbon in the methyl isobutyl ketone molecule, which increases the negative charge on the oxygen atom of the carbonyl group. The negative $V^{\mathrm{E}}$ values for the system that contains cyclopentanone may be attributed to the small ring structured cyclopentanone molecules being easily accommodated interstitially in the void space of $N$-methyl aniline. The shape and size of the ketones and their cosolvent are not similar. So, on mixing of the solvents, non-specific physical interactions and unfavorable interactions between unlike component molecules come into play thereby increasing the volume of binary solvent mixtures.

The positive $V^{\mathrm{E}}$ values for the systems that contain cyclohexanone may be attributed to the ring structured $\mathrm{CH}$ molecules rupturing the hydrogen bonding in $\mathrm{N}$-methyl aniline. 


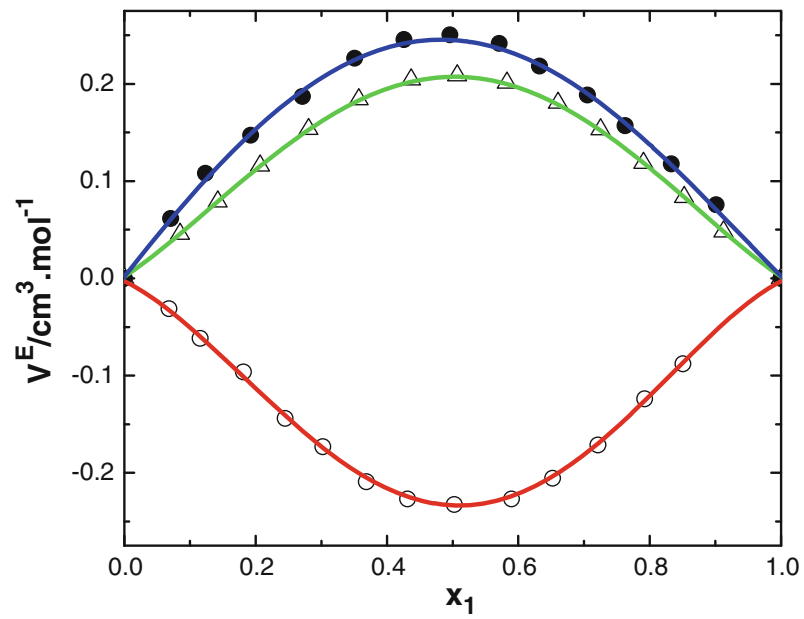

Fig. 8 Variation of excess molar volume $\left(V^{\mathrm{E}}\right)$ with mole fraction $\left(x_{1}\right)$ of N-MA in the binary liquid mixtures of N-MA with cyclopentanone (small circle), 2-methyl cyclohexanone (small triangle) and cyclohexanone (small black circle) at $303.15 \mathrm{~K}$

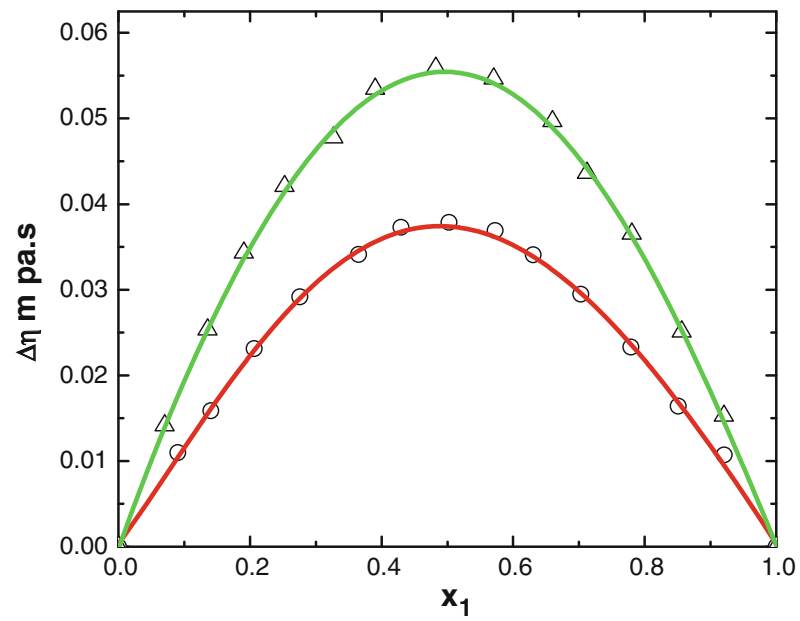

Fig. 9 Deviation of viscosity $\Delta \eta$ with mole fraction $\left(x_{1}\right)$ of N-MA in the binary liquid mixtures of N-MA with methyl isobutyl ketone (small circle) and diethyl ketone (small triangle) at $303.15 \mathrm{~K}$

Furthermore, interactions of $\mathrm{N}$-methyl aniline with methyl cyclohexanone (Me-CH) may be ascribed to the methyl group of $\mathrm{Me}-\mathrm{CH}$.

The positive excess volume data of $\mathrm{Me}-\mathrm{CH}$ is less than that of $\mathrm{CH}$ due to the positive inductive effect of the methyl group.

The $V^{\mathrm{E}}$ values of N-MA with ketones follow the order: $\mathrm{MIBK}<\mathrm{DEK}<\mathrm{CP}<\mathrm{Me}-$ $\mathrm{CH}<\mathrm{CH}$.

The deviation in viscosity $(\Delta \eta)$ of all the binary mixtures are graphically represented in Figs. 9 and 10. The sign and magnitude of deviation in viscosity may depend on the 


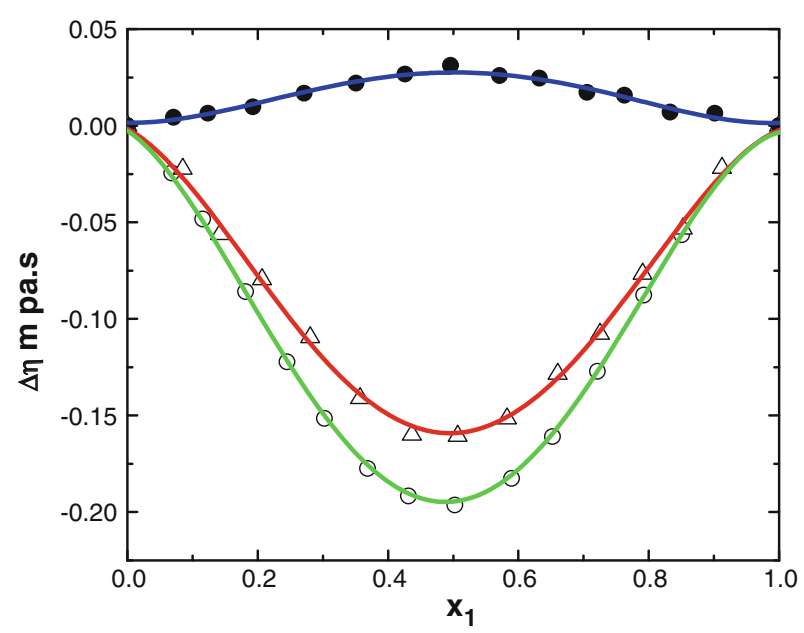

Fig. 10 Deviation of viscosity $\Delta \eta$ with mole fraction $\left(x_{1}\right)$ of N-MA in the binary liquid mixtures of N-MA with cyclopentanone (small circle), 2-methyl cyclohexanone (small triangle) and cyclohexanone (small black circle) at $303.15 \mathrm{~K}$

combined effect of factors such as molecular size, shape and intermolecular forces [30]. In general for the systems where dispersion and dipolar interactions are operating, $\Delta \eta$ values are found to be negative [31-33], whereas charge transfer, hydrogen bonding interactions and other chemical forces lead to the formation of complex species between unlike component molecules that result in positive values of $\Delta \eta$. The actual values depend upon the dominant factor [34]. An examination of data in the Table 4 shows that the values of $\Delta \eta$ for the systems $\mathrm{N}-\mathrm{MA}+\mathrm{MIBK}, \mathrm{N}-\mathrm{MA}+\mathrm{DEK}$ and $\mathrm{N}-\mathrm{MA}+\mathrm{CH}$ are positive whereas for the mixtures of N-MA + CP and N-MA + Me-CH they are negative over the entire composition ranges at $303.15 \mathrm{~K}$.

The $\Delta \eta$ values for the systems containing methyl isobutyl ketone, diethyl ketone and alicyclic ketones fall in the order: methyl isobutyl ketone $<$ diethyl ketone and cyclopentanone $<$ methylcyclohexanone $<$ cyclohexanone (Fig. 8).

According to Reed and Taylor [35] positive deviations in $G^{* \mathrm{E}}$ may be due to specific interactions like hydrogen bonding and charge transfer, whereas the negative deviations may be ascribed to dispersion forces within the systems. An examination of data in the Table 3 suggests that the values of $G^{*}$ for the systems N-MA $+\mathrm{MIBK},+\mathrm{DEK},+\mathrm{CH}$ are positive whereas for the mixtures of $\mathrm{N}-\mathrm{MA}+\mathrm{CP},+\mathrm{Me}-\mathrm{CH}$ are negative over the entire composition ranges at $303.15 \mathrm{~K}$. In the present investigation, positive values of $G^{* \mathrm{E}}$ may be attributed to dipole-dipole interactions between the component molecules and the negative values show the dispersion forces. Recently, Ali et al. attributed the positive values of $G^{* \mathrm{E}}$ in liquid mixtures to hydrogen bond formation between unlike molecules.

The interaction parameter $d$, in the Grunberg and Nissan equation, is a measure of the strength of interaction between the mixing components. Table 4 shows that the values of $d$ are negative for the systems: $N$-methyl aniline + cyclopentanone and $N$-methyl aniline + methylcyclohexanone and are positive for the systems: $N$-methyl aniline + methyl isobutyl ketone, $\mathrm{N}$-methyl aniline + diethyl ketone, and $\mathrm{N}$-methyl aniline + cyclohexanone at $303.15 \mathrm{~K}$. According to Kalra et al. [36], large and positive $d$ values indicate strong specific interaction, small positive values indicate weak specific interaction and large 
Table 4 Experimental and calculated values of viscosity $(\eta)$, deviation in viscosities $(\Delta \eta)$, excess Gibbs energy of activation of viscous flow $\left(G^{* \mathrm{E}}\right)$, Grunberg-Nissan interaction parameters $(d)$, Katti-Chaudhri interaction parameters $\left(W_{\text {vis }} / R T\right)$, and Hind interaction parameters $\left(H_{12}\right)$ at $303.15 \mathrm{~K}$

\begin{tabular}{|c|c|c|c|c|c|c|}
\hline$x_{1}$ & $\eta(\mathrm{mPa} \cdot \mathrm{s})$ & $\Delta \eta$ (mPa.s) & $G^{* \mathrm{E}}\left(\mathrm{J} \cdot \mathrm{mol}^{-1}\right)$ & $d$ & $W_{\text {vis }} / R T$ & $H_{12}$ \\
\hline \multicolumn{7}{|c|}{$N-M A(1)+$ methylisobutylketone (2) } \\
\hline 0.0000 & 0.525 & 0.000 & 0.000 & & & \\
\hline 0.0901 & 0.645 & 0.011 & 0.107 & 0.520 & 0.520 & 1.197 \\
\hline 0.1406 & 0.711 & 0.015 & 0.148 & 0.486 & 0.485 & 1.196 \\
\hline 0.2065 & 0.798 & 0.023 & 0.188 & 0.456 & 0.454 & 1.201 \\
\hline 0.2759 & 0.888 & 0.029 & 0.213 & 0.426 & 0.424 & 1.203 \\
\hline 0.3652 & 1.001 & 0.034 & 0.227 & 0.391 & 0.389 & 1.204 \\
\hline 0.4295 & 1.082 & 0.037 & 0.228 & 0.372 & 0.369 & 1.206 \\
\hline 0.5026 & 1.171 & 0.037 & 0.219 & 0.350 & 0.347 & 1.206 \\
\hline 0.5728 & 1.255 & 0.036 & 0.203 & 0.332 & 0.329 & 1.205 \\
\hline 0.6305 & 1.322 & 0.034 & 0.184 & 0.317 & 0.314 & 1.203 \\
\hline 0.7029 & 1.405 & 0.029 & 0.157 & 0.300 & 0.297 & 1.201 \\
\hline 0.7791 & 1.491 & 0.023 & 0.122 & 0.284 & 0.282 & 1.198 \\
\hline 0.8509 & 1.571 & 0.016 & 0.086 & 0.270 & 0.269 & 1.195 \\
\hline 0.9209 & 1.650 & 0.010 & 0.049 & 0.264 & 0.264 & 1.204 \\
\hline 1.0000 & 1.735 & 0.000 & 0.000 & & & \\
\hline \multicolumn{7}{|c|}{ N-MA (1) + diethylketone (2) } \\
\hline 0.0000 & 0.425 & 0.000 & 0.000 & & & \\
\hline 0.0701 & 0.531 & 0.014 & 0.135 & 0.827 & 0.823 & 1.189 \\
\hline 0.1356 & 0.628 & 0.025 & 0.217 & 0.740 & 0.736 & 1.188 \\
\hline 0.1906 & 0.709 & 0.034 & 0.265 & 0.686 & 0.681 & 1.191 \\
\hline 0.2526 & 0.798 & 0.042 & 0.298 & 0.632 & 0.627 & 1.191 \\
\hline 0.3269 & 0.901 & 0.047 & 0.316 & 0.575 & 0.570 & 1.189 \\
\hline 0.3905 & 0.990 & 0.053 & 0.321 & 0.541 & 0.535 & 1.192 \\
\hline 0.4825 & 1.113 & 0.055 & 0.307 & 0.494 & 0.489 & 1.192 \\
\hline 0.5705 & 1.227 & 0.054 & 0.279 & 0.457 & 0.451 & 1.192 \\
\hline 0.6598 & 1.339 & 0.049 & 0.237 & 0.425 & 0.419 & 1.191 \\
\hline 0.7125 & 1.402 & 0.043 & 0.207 & 0.406 & 0.401 & 1.186 \\
\hline 0.7805 & 1.484 & 0.036 & 0.165 & 0.387 & 0.382 & 1.187 \\
\hline 0.8564 & 1.572 & 0.025 & 0.112 & 0.365 & 0.361 & 1.182 \\
\hline 0.9204 & 1.646 & 0.015 & 0.064 & 0.352 & 0.348 & 1.184 \\
\hline 1.0000 & 1.735 & 0.000 & 0.000 & & & \\
\hline \multicolumn{7}{|c|}{$N-M A(1)+$ cyclopentanone $(2)$} \\
\hline 0.0000 & 0.996 & 0.000 & 0.000 & & & \\
\hline 0.0682 & 1.022 & -0.024 & -0.012 & -0.082 & -0.075 & 1.173 \\
\hline 0.1154 & 1.033 & -0.048 & -0.028 & -0.117 & -0.110 & 1.129 \\
\hline 0.1812 & 1.044 & -0.086 & -0.056 & -0.156 & -0.150 & 1.076 \\
\hline 0.2451 & 1.055 & -0.122 & -0.083 & -0.184 & -0.178 & 1.035 \\
\hline 0.3024 & 1.068 & -0.151 & -0.104 & -0.201 & -0.196 & 1.006 \\
\hline 0.3687 & 1.091 & -0.177 & -0.121 & -0.211 & -0.206 & 0.984 \\
\hline 0.4312 & 1.123 & -0.192 & -0.127 & -0.211 & -0.206 & 0.974 \\
\hline 0.5025 & 1.171 & -0.196 & -0.125 & -0.203 & -0.198 & 0.972 \\
\hline
\end{tabular}


Table 4 continued

\begin{tabular}{|c|c|c|c|c|c|c|}
\hline$x_{1}$ & $\eta(\mathrm{mPa} \cdot \mathrm{s})$ & $\Delta \eta$ (mPa.s) & $G^{* \mathrm{E}}\left(\mathrm{J} \cdot \mathrm{mol}^{-1}\right)$ & $d$ & $W_{\mathrm{vis}} / R T$ & $H_{12}$ \\
\hline 0.5894 & 1.249 & -0.183 & -0.107 & -0.180 & -0.176 & 0.988 \\
\hline 0.6521 & 1.317 & -0.161 & -0.087 & -0.158 & -0.153 & 1.010 \\
\hline 0.7214 & 1.402 & -0.127 & -0.061 & -0.126 & -0.121 & 1.049 \\
\hline 0.7925 & 1.494 & -0.088 & -0.035 & -0.090 & -0.085 & 1.099 \\
\hline 0.8504 & 1.568 & -0.056 & -0.018 & -0.062 & -0.056 & 1.143 \\
\hline 1.0000 & 1.735 & 0.000 & 0.000 & & & \\
\hline \multicolumn{7}{|c|}{$N$-MA (1) + 2-methylcyclohexanone (2) } \\
\hline 0.0000 & 2.225 & 0.000 & 0.000 & & & \\
\hline 0.0851 & 2.161 & -0.022 & -0.007 & -0.045 & -0.040 & 1.836 \\
\hline 0.1425 & 2.099 & -0.056 & -0.023 & -0.081 & -0.076 & 1.750 \\
\hline 0.2069 & 2.044 & -0.079 & -0.034 & -0.088 & -0.083 & 1.737 \\
\hline 0.2804 & 1.978 & -0.109 & -0.049 & -0.103 & -0.097 & 1.708 \\
\hline 0.3569 & 1.909 & -0.141 & -0.067 & -0.122 & -0.116 & 1.672 \\
\hline 0.4368 & 1.851 & -0.160 & -0.079 & -0.133 & -0.127 & 1.654 \\
\hline 0.5069 & 1.816 & -0.160 & -0.080 & -0.134 & -0.128 & 1.658 \\
\hline 0.5824 & 1.788 & -0.151 & -0.077 & -0.132 & -0.126 & 1.668 \\
\hline 0.6602 & 1.773 & -0.128 & -0.065 & -0.122 & -0.116 & 1.693 \\
\hline 0.7251 & 1.762 & -0.107 & -0.055 & -0.115 & -0.109 & 1.709 \\
\hline 0.7905 & 1.761 & -0.076 & -0.038 & -0.098 & -0.092 & 1.748 \\
\hline 0.8526 & 1.754 & -0.053 & -0.026 & -0.089 & -0.083 & 1.768 \\
\hline 0.9125 & 1.756 & -0.021 & -0.009 & -0.053 & -0.047 & 1.843 \\
\hline 1.0000 & 1.735 & 0.000 & 0.000 & & & \\
\hline \multicolumn{7}{|c|}{$N-M A(1)+$ cyclohexanone $(2)$} \\
\hline 0.0000 & 1.812 & 0.000 & 0.000 & & & \\
\hline 0.0705 & 1.811 & 0.004 & 0.003 & 0.016 & 0.021 & 1.807 \\
\hline 0.1236 & 1.809 & 0.006 & 0.005 & 0.014 & 0.019 & 1.803 \\
\hline 0.1924 & 1.807 & 0.009 & 0.007 & 0.015 & 0.020 & 1.805 \\
\hline 0.2714 & 1.808 & 0.016 & 0.012 & 0.021 & 0.025 & 1.816 \\
\hline 0.3508 & 1.807 & 0.022 & 0.016 & 0.023 & 0.028 & 1.821 \\
\hline 0.4258 & 1.806 & 0.026 & 0.019 & 0.027 & 0.031 & 1.828 \\
\hline 0.4957 & 1.805 & 0.031 & 0.022 & 0.030 & 0.035 & 1.835 \\
\hline 0.5708 & 1.794 & 0.026 & 0.018 & 0.026 & 0.030 & 1.826 \\
\hline 0.6321 & 1.788 & 0.024 & 0.017 & 0.026 & 0.030 & 1.826 \\
\hline 0.7054 & 1.775 & 0.017 & 0.013 & 0.020 & 0.025 & 1.815 \\
\hline 0.7625 & 1.769 & 0.015 & 0.011 & 0.021 & 0.025 & 1.816 \\
\hline 0.8327 & 1.755 & 0.007 & 0.005 & 0.013 & 0.016 & 1.799 \\
\hline 0.9015 & 1.749 & 0.006 & 0.005 & 0.018 & 0.022 & 1.809 \\
\hline 1.0000 & 1.735 & 0.000 & 0.000 & & & \\
\hline
\end{tabular}

negative values indicate no specific interaction. Hence, the negative values of $d$ may be attributed to the dominance of dispersion forces arising from the breaking of hydrogen bonds in the associated component of the mixtures and positive $d$ values due to specific interactions. 


\section{Conclusions}

In this paper, the densities, viscosities and speed of sound at $303.15 \mathrm{~K}$ have been measured over the entire range of composition of $N$-methyl aniline with ketones. From these measured physico-chemical data, excess molar volumes, deviation in viscosities, deviation in ultrasonic sound velocities and excess isentropic compressibility have been calculated. These data were correlated by a Redlich-Kister type polynomial equation to derive the coefficients and standard deviation. The results are interpreted in terms of molecular interactions between the component molecules.

Acknowledgments The author M. Gowrisankar is grateful to SERO (U.G.C) Hyderabad for awarding a Teacher Fellowship and expresses gratitude to the management of J.K.C. College, K.V.N.B. Kumar, Principal, J.K.C. College, Guntur - 522006, for giving permission and encouragement and also expresses gratitude to the Department of Chemistry, S.V. University, Tirupathi for being allowed to carry out the present research work.

Open Access This article is distributed under the terms of the Creative Commons Attribution License which permits any use, distribution, and reproduction in any medium, provided the original author(s) and the source are credited.

\section{References}

1. Ali, A., Nain, A.K., Kamil, M.: Physico-chemical studies of non-aqueous binary liquid mixtures at various temperatures. Thermochim. Acta 274, 209-221 (1996)

2. Grunberg, L., Nissan, A.H.: Mixture law for viscosity. Nature 164, 799-800 (1949)

3. Katti, P.K., Chaudhri, M.H.: Viscosities of binary mixtures benzyl acetate with dioxane, aniline and $m$ cresol. J. Chem. Eng. Data 9, 442-443 (1964)

4. Hind, R.K., McLaughlin, E., Ubbelohde, A.R.: Structure and viscosity of liquid camphor and pyrene mixtures. Trans. Faraday Soc. 56, 328-330 (1960)

5. Gowri Sankar, M., Sivarambabu, S., Venkateswarlu, P., Siva Kumar, K.: Excess volumes, speeds of sound, isentropic compressibilities and viscosities of binary mixtures of $N$-ethyl aniline with some aromatic ketones at 303.15 K. Bull. Korean Chem. Soc. 33, 1686-1692 (2012)

6. Gowri Sankar, M., Venkateswarlu, P., Siva Kumar, K., Sivarambabu, S.: Thermodynamics of amine + ketone mixtures 3 . Volumetric, speed of sound data and viscosity at (303.15 and $308.15 \mathrm{~K}$ ) for the binary mixtures of $N, N$-dimethylaniline + propiophenone, $+p$-methylacetophenone, $+p$-chloroacetophenone. J. Mol. Liq. 173, 172-179 (2012)

7. Alonso, I., Alonso, V., Muzo, I., Garcia de la Fuente, I., Gonzalez, J.A., Cubos, J.C.: Thermodynamics of ketone + amine mixtures, I. Volumetric and speed of sound data at (293.15, 298.15 and 303.15) K for 2-propanone + aniline, $+N$-methylaniline, or + pyridine systems. J. Chem. Eng. Data 55, 2505-2511 (2010)

8. Alonso, I., Alonso, V., Muzo, I., Garcia de la Fuente, I., Gonzalez, J.A., Cubos, J.C.: Thermodynamics of ketone + amine mixtures, Part III. Volumetric and speed of sound data at $(293.15,298.15$ and 303.15) $\mathrm{K}$ for 2-butanone + aniline, $+N$-methylaniline, or + pyridine systems. J. Chem. Eng. Data 55, 5400-5405 (2010)

9. Alonso, I., Alonso, V., Muzo, I., Garcia de la Fuente, I., Gonzalez, J.A., Cubos, J.C.: Thermodynamics of ketone + amine mixtures, 7. Volumetric and speed of sound data at $(293.15,298.15$ and 303.15) K for 2-pentanone + aniline, $+N$-methylaniline, or + pyridine systems. J. Mol. Liq. 160, 180-186 (2011)

10. Riddick, J.A., Bunger, W.B., Sakano, T.K.: Organic Solvents-Physical Properties and Method of Purifications, vol. 2. Wiley Interscience, New York (1986)

11. Palepu, R., Diver, J., Campell, D.: Thermodynamic and transport properties of $o$-chlorophenol with aniline and $N$-alkyl anilines. J. Chem. Eng. Data 30, 355-360 (1985)

12. Comelli, F., Francesconic, R.: Densities and excess molar volumes of propylene carbonate + linear and cyclic ketones at 298.15 K. J. Chem. Eng. Data 40, 808-810 (1995)

13. Fermeglla, M., Romano, L., Glovanni, T.: Excess volumes and viscosities of binary systems containing 4-methyl-2-pentanone. J. Chem. Eng. Data 35, 260-265 (1990) 
14. Oswal, S.L., Pandiyan, V., Krishnakumar, B., Vasantharani, P.: Thermodynamic and acoustic properties of binary mixtures of oxolane with aniline and substituted anilines at $303.15,313.15$ and $323.15 \mathrm{~K}$. Thermochim. Acta 507, 27-34 (2010)

15. Radhamma, M., Venkatesu, P., Prabhakara Rao, M.V., Lee, M.-J., Lin, H.-M.: Excess molar volumes and ultrasonic studies of dimethylsulphoxide with ketones at $T=303.15 \mathrm{~K}$. J. Chem. Thermodyn. 40, 492-497 (2008)

16. Dharmaraju, G., Narayanaswamy, G., Raman, G.K.: Excess volumes and isentropic compressibilities of binary mixtures of a ketone and acetonitrile. J. Chem. Eng. Data 27, 193-195 (1982)

17. Lesbats, C., Lichanot, A.: Capacities calorifiques de durcisseurs amines et resins epoxydes. Thermochim. Acta 109, 317-329 (1987)

18. Rathnam, M.V., Sudhir, M., Kumar, M.S.: Densities, viscosities and refractives indices of binary mixtures of diethyl oxalate with some ketones at (303.15, 308.15 and 303.15) K. J. Chem. Eng. Data 55, 5946-5952 (2010)

19. Fuchs, R.: Heat capacities of ketones and aldehydes at 298.15 K. Can. J. Chem. 58, 2305-2307 (1980)

20. Jaganath, D.A.P.: Ultrasonic velocities in, and adiabatic compressibilities and excess volumes for, binary mixtures of acetone with tetrachloroethane, trichloroethylene, methylene chloride, 1,2-dichloromethane, and cyclohexane. J. Chem. Eng. Data 29, 313-316 (1984)

21. Krishnaiah, A., Surendranath, K.N.: Densities, speeds of sound and viscosities of mixtures of oxolane with chloroethanes and chloroethenes. J. Chem. Eng. Data 41, 1012-1014 (1996)

22. Jacobson, B.: Ultrasonic velocity of liquids and liquids mixtures. J. Chem. Phys. 20, 927-928 (1952)

23. Benson, G.C., Kiyohara, O.: Evaluation of excess isentropic compressibilities and isochoric heat capacities. J. Chem. Thermodyn. 11, 1061-1064 (1979)

24. Redlich, O., Kister, A.T.: Thermodynamics of non electrolytic solutions. Algebraic representation of thermodynamic properties and the classification of solutions. Ind. Eng. Chem. 40, 345-348 (1948)

25. Kumar Nain, A.: Ultrasonic and viscometric studies of molecular interactions in binary mixtures of formamide with ethanol, 1-propanol, 1,2-ethanediol and 1,2-propanediol at different temperatures. J. Mol. Liq. 140, 108-116 (2008)

26. Kawaizumi, F., Ohno, M., Miyahara, Y.: Ultrasonic and volumetric investigation of aqueous solutions of amides. Bull. Chem. Soc. Jpn. 50, 2229-2233 (1977)

27. Sri Devi, U., Samatha, K., Visvanantasarma, A.: Excess thermodynamic properties in binary liquids. J. Pure Appl. Ultras. 26, 1-11 (2004)

28. Fort, R.J., Moore, W.R.: Adiabatic compressibilities of binary liquids mixtures. Trans. Faraday Soc. 62, 2102-2111 (1965)

29. Kumar, H., Chahal, S.: Studies of some thermodynamic properties of binary mixtures of acrylonitrile with aromatic ketones at $T=308.15$ K. J. Solution Chem. 40, 165-181 (2011)

30. Nath, J., Srivastava, A.K.: Excess volumes for binary liquid mixtures of trichloroethylene with anisole, pyridine, quinoline and cyclohexane at 298.15 and 308.15 K. Fluid Phase Equilib. 28, 97-101 (1986)

31. Venkatesu, P., Chandra Sekhar, G., Prabhakar Rao, M.V.: Ultrasonic studies of $N, N$-dimethyl formamide + cyclohexanone + 1-alkanols at 303.15 K. Phys. Chem. Liq. 44, 287-291 (2006)

32. Pikkarainam, L.: Densities and viscosities of binary mixtures of $N, N$-dimethyl acetamide with aliphatic alcohols. J. Chem. Eng. Data 28, 344-347 (1983)

33. Fort, R.J., Moore, W.R.: Viscosities of binary liquids mixtures. Trans. Faraday Soc. 62, 1112-1119 (1966)

34. Jyostna, T.S., Satyanarayana, N.: Density and viscosity of binary liquid systems of $N$-methylacetamide with aromatic ketones at $T=308.15$ K. J. Chem. Thermodyn. 38, 272-277 (2006)

35. Reed III, T.M., Taylor, T.E.: Viscosity of liquid mixtures. J. Phys. Chem. 63, 58-67 (1959)

36. Kalra, K.C., Singh, K.C., Spah, D.C.: Viscosities of 1,2-dibromoethane + aromatic hydrocarbon mixtures and prediction-of excess thermodynamic functions. J. Ind. Chem. Soc. 69, 138-141 (1992) 